\title{
Visualization of Complex Networks Dynamics: Case Study
}

\author{
Ivan Zelinka, Donald Davendra, and Lenka Skanderova \\ Department of Computer Science, \\ Faculty of Electrical Engineering and Computing Science, \\ Technical University of Ostrava, Tr. 17. Listopadu 15, Ostrava, \\ Czech Republic \\ \{ivan.zelinka, donald. davendra, lenka.skanderova.st\}@vsb.cz
}

\begin{abstract}
In this article is discussed novel method of the so-called complex networks dynamics and its visualization by means of so called coupled map lattices method. The main aim of this research is to demonstrate possibility to visualize complex network dynamics by means of the same method, that is used spatiotemporal chaos modeling. It is suggested here to use coupled map lattices system to simulate complex network so that each site is equal to one vertex of complex network. Interaction between network vertices is in coupled map lattices equal to the strength of mutual influence between system sites. Also another results from previous experiments, where dynamics of evolutionary algorithms has been converted to complex network and consequently to CML, are mentioned at the end. All results has been properly visualized and explained.
\end{abstract}

Keywords: complex networks, network dynamics, coupled map lattices, chaos, and visualization, control of complex systems.

\section{Introduction}

In this article, we try to merge two completely different (at first glance) areas of research: complex networks and chaotic systems visualization.

Large-scale networks, exhibiting complex patterns of interaction amongst vertices exist in both nature and in man-made systems (i.e., communication networks, genetic pathways, ecological or economical networks, social networks, networks of various scientific collaboration, Internet, World Wide Web, power grid etc.). The structure of complex networks thus can be observed in many systems.

The word "complex" networks [1], [2] comes from the fact that they exhibit substantial and non-trivial topological features, with patterns of connection between vertices that are neither purely regular nor purely random. Such features include a heavy tail in the degree distribution, a high clustering coefficient, and hierarchical structure, amongst other features. In the case of directed networks, these features also include reciprocity, triad significance profile and other features. 
As an example of another exhibition of the existence of complex networks are evolutionary algorithms, whose dynamics can be visualized like complex network structure and dynamics.

The main idea of our research is to show in this article that the dynamics of complex network can be analyzed and visualized like a coupled map lattices (CML), which is usually used for visualization of the chaotic systems. CML system can be understood like a row (1D case) or field of $\mathrm{N} \times \mathrm{M}$ mechanical oscillators, which are mutually joined and influent themselves via nonlinear coupling parameter $\varepsilon$. Their behavior is then visualized as done in Figure 1. Different levels of colors (or levels of gray) represent different "energy" (phase) of each oscillator.

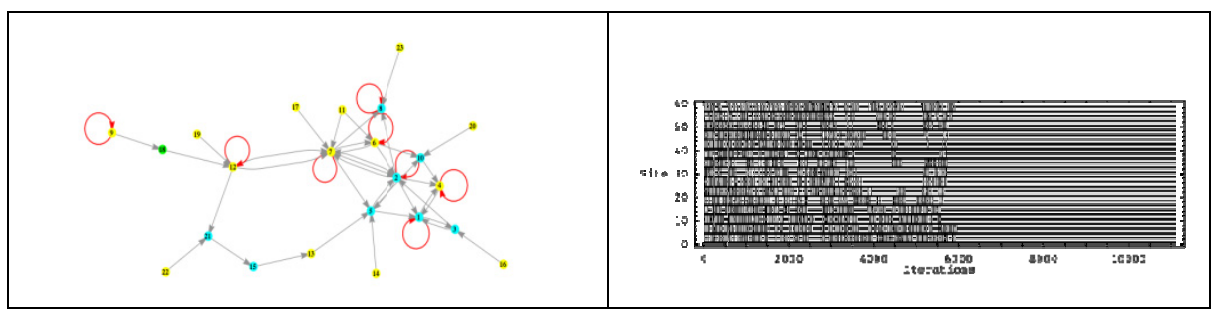

Fig. 1. Example: complex network with more vertices (left), typical CML behavior with chaotic and deterministic "windows", after iteration 6000 it is due to the control fully stabilized (right)

Complex network science is very popular in the last 10 years. Root of it comes from social complex networks, like citation networks etc. During the time it become to be very popular and interdisciplinary important scientific field of research. It has been discovered, that complex network structure and its dynamics, is hidden in the background of many processes that are not, on the first glance, related to the complex networks. Lets note for example classical examples like citation networks, social networks kind of facebook, interactions on the stock exchange, complex communication or power networks, interactions and stress level between people in the closed groups (space flights, submarine missions) etc. are. Another side of coin we can note (from our research) complex network, that is hidden behind dynamics of evolutionary algorithms, see Figure 3 and for full report [3]. This is typical example of formed complex network that show interactions between individuals in the population of the evolutionary algorithm. Each node on Figure 3 is in fact individual from population and links amongst them represent its activities (i.e. which individual has been selected to be parent etc.).

Based on fact, that complex networks and its dynamics can be discovered almost everywhere today, question how to control, analyze or predict them is of course important one. Of course, a lot of standard techniques exist today there. All those techniques, see for example [4], are based on classical mathematical tools, or on heuristic methods, see [5], [6] and [7]. As mentioned before, our aim in this article is to show, that there is quite simple approach how to visualize complex networks and its growth 
and dynamic in the time so, that well-known methods from chaos control can be used. This approach is discussed in the next section.

\section{Complex Networks Dynamics and Its Vizualization}

Our method of visualization is based on fact that simplest version of CML (i.e. 1D version) is usually depicted like a row of mutually joined sites, where each site is nonlinearly joined with its nearest sites. Our vision of equivalence between CML and complex network is quite simple. Each vertex is equivalent to the site in the CML. Comparing to the standard CML, sites in complex network CML (CNCML) are not joined to the nearest site, but to the sites equal to the complex network vertices. Thus sites in CNCML are not joined symmetrically (i.e. from site $\mathrm{X}$ to $\mathrm{Y}$ and vice versa) and between different sites is random pattern of connections, which can change in the time. Lets see as an example Figure 1. Vertex No. 12 have feedback to itself (red selfloop), then it influent vertices 7 (double edge), 21 and are influenced by vertices (CNCML sites) 18 and 19. As it is visible, based on our interpretation ( $\mathrm{CN}=$ CNCML), CNCML is more complex version of the classical simplified version of the CML, however still CML in general, so all techniques of control and analysis shall be working on such a version of CML. Our experiments of CNCML visualization were based on approach that on the beginning has been generated complex network with big number of vertices and no another vertex has been added after all. Only weights on the randomly selected edges have been modified. In all cases has been CNCML calculated and visualized in such a way, that for each $\mathrm{CN}$ vertex (i.e. CNCML site) has been calculated according to (5). Different levels of vertices (sites) excitation are depicted by different colors. When compared with Figure 2, it is clearly visible, that our proposed kind of visualization is usable. In Figure 2 and Figure 3 it is observable, that CNCML visualization shows complex and obviously nonlinear behavior of tested CNs. Different kind of behavior (different colors) is clearly visible. Dynamics of the complex network with edges deactivation by real number coefficient. Different colors (gray levels) including white one represent different vertices (sites) activation.

$$
\begin{aligned}
& S_{k}=\sum_{i=1}^{N} w_{j} \\
& \text { where } \\
& S_{k} \ldots \text { is state (excitation or inhibition) of } K^{. t} \mathrm{CN} \text { vertex (CNCML site) } \\
& \quad N \ldots \text { is number of CN vertices } \\
& w_{i} \ldots \text { is weight (random number in general) associated to the each edge. } \\
& \quad \text { If } L^{* k} \text { edge does not exist, then } w_{L}=0
\end{aligned}
$$




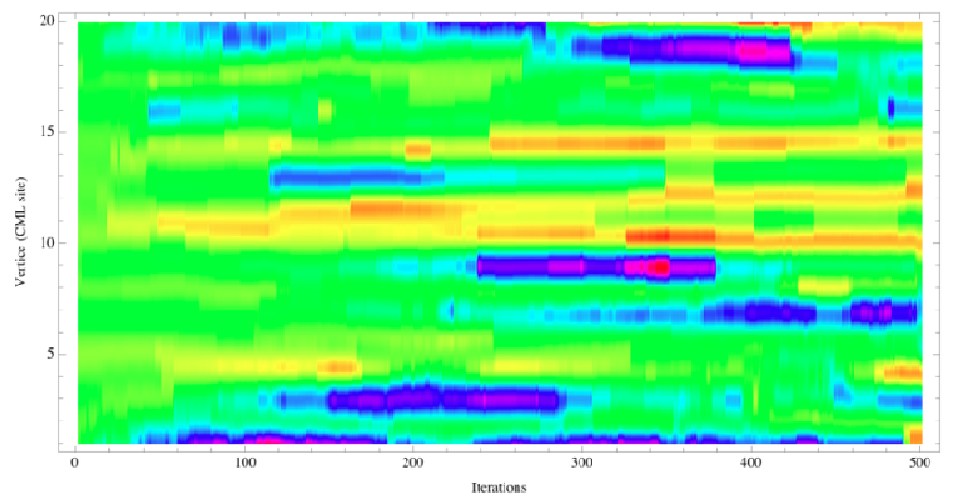

Fig. 2. CML of the network with 20 vertices in 500 iterations

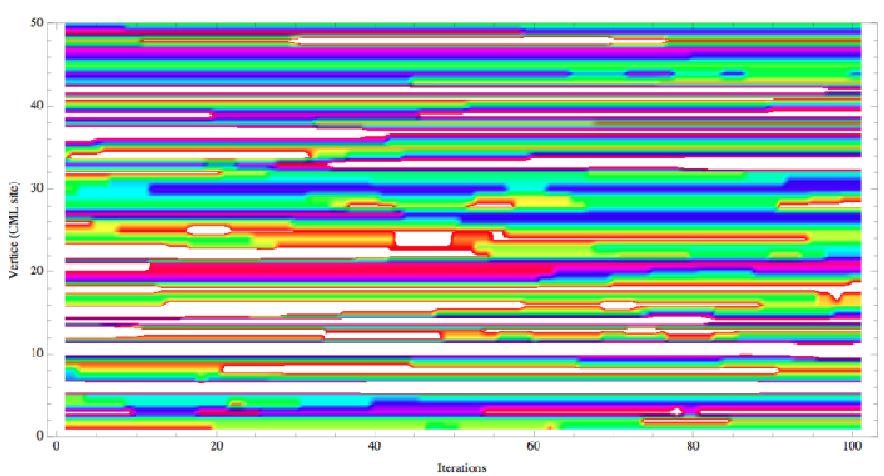

Fig. 3. Dynamics of the complex network visualized like CML system with edges deactivation by real number coefficient. Different colors including white one represent different vertices (sites) activation.

\section{Conclusions}

In this paper we have proposed method how to visualize and simulate (model) complex network dynamics by means of the CML systems. CML modeling is well known in the domain of so-called spatiotemporal deterministic chaos. For analysis and control of the CML systems has been developed numerous techniques, based on classical mathematics as well as on the heuristic methods. Based on this fact we have used and tested method, how to visualize $\mathrm{CN}$ dynamics as a CML system. As a summarization and conclusion can be state this:

- The model of complex network, used for simulations here, has been based on randomly initialized network with different number of vertices.

- Edges between vertices have been also initialized randomly, however, roulette method from genetic algorithms, have been used to add the new edge to the vertex, and/or increase or decrease importance of existing edge. Roulette 
method has been used in order to prefer richer vertices (i.e. vertices with more incoming edges) and thus support in this way the main idea of complex networks with small world phenomenon.

- One kind of dynamics for $\mathrm{CN}$ visualization has been used. It has been based on preliminary fact, that we already have developed network, with constant number of vertices and dynamic itself depends only on the edge weights evolution.

- All substantial and typical results are visualized via Figure 3 - Figure 2 and it can be stated that our technique of CNCML is usable and complex behavior of $\mathrm{CN}$ can be visualized in this way. It is also obvious, that methods of analysis and control of CMLs can be also used for CNCML, because conversion classical CN to the CNCML basically create specific, more complex version, of the CML and there are no restriction of use of contemporary methods on such a version of CML. On the other side, in the case of big computational complexity for complex and big CNCMLs, heuristics, like evolutionary algorithms [8], [5], [6], [7] can be easily used.

Another, more advanced results of this approach are represented in [9]. The main motivation of this research is whether it is possible to visualize and simulate underlying dynamics of an evolutionary process as a complex network. Based on preliminary results (based only on 2 algorithms in 10 versions and 16 test function out of 17) it can be stated that its possible use and benefit of this approach is according to us novel approach to evaluate and control evolutionary dynamics. Based on numerically demonstrated fact (no mathematical proof has been made) that EAs dynamics can be visualized like complex networks we believe that there is new research area for study of EAs dynamics and its possible control via techniques of complex network control [4]. The next research shall be focused on collection of the data from existing real CNs, based on [9] also exact analysis, when chaotic regimes are observable in CNCML, will be done and also what kind of routes to chaos (intermittence, crisis, bifurcations) are there shall be interesting. Another open research issue is to use of selected evolutionary algorithms on CNCML control with "reverse" investigation, what exact impact has such a control on the original $\mathrm{CN}$ structure (basically control here means that EAs have to change structure of $\mathrm{CN}$ ).

Acknowledgement. This work was supported by IT4Innovations Centre of Excellence project, reg. no. CZ.1.05/1.1.00/02.0070 supported by Operational Program 'Research and Development for Innovations' funded by Structural Funds of the European Union and state budget of the Czech Republic.

\section{References}

1. Dorogovtsev, S.N., Mendes, J.F.F.: Evolution of Networks. Adv. Phys. 51, 1079 (2002)

2. Boccaletti, S., et al.: Complex Networks: Structure and Dynamics. Phys. Rep. 424, 175-308 (2006) 
3. Zelinka, I., Davendra, D.: Investigation on Relations Between Complex Networks and Evolutionary Algorithm Dynamics. International Journal of Computer Information Systems and Industrial Management Applications (2011) (in print)

4. Meyn, S.: Control Techniques for Complex Networks. Cambridge University Press (2007)

5. Zelinka, I.: Investigation on Realtime Deterministic Chaos Control by Means of Evolutionary Algorithms, In: 1st IFAC Conference on Analysis and Control of Chaotic Systems, Reims, France (2006)

6. Senkerik, R., Zelinka, I., Navratil, E.: Optimization of feedback control of chaos by evolutionary algorithms. In: 1st IFAC Conference on Analysis and Control of Chaotic Systems, Reims, France (2006)

7. Zelinka, I.: Investigation on real-time deterministic chaos control by means of evolution-ary algorithms. In: Proc. First IFAC Conference on Analysis and Control of Chaotic Systems, Reims, France, pp. 211-217 (2006)

8. Zelinka, I.: Real-time deterministic chaos control by means of selected evolutionary algorithms. Engineering Applications of Artificial Intelligence (2008), doi:10.1016/j.engappai.2008.07.008

9. Zelinka, I., Davendra, D., Senkerik, R., Jasek, R.: Does Evolutionary Algorithms Dynamics Create a Complex Networks Structures? Complex Systems 20(2), Champaign, IL 61826 USA 